American Journal of Applied Sciences, 10 (4): 307-312, 2013

ISSN: 1546-9239

(C) 2013 W.A. Rahoma, This open access article is distributed under a Creative Commons Attribution

(CC-BY) 3.0 license

doi:10.3844/ajassp.2013.307.312 Published Online 10 (4) 2013 (http://www.thescipub.com/ajas.toc)

\title{
Lunisolar Invariant Relative Orbits
}

\author{
Walid Ali Rahoma \\ Department of Astronomy and Space Science, Faculty of Science, Cairo University, 12613, Egypt
}

Received 2012-05-07, Revised 2012-09-19; Accepted 2013-04-24

\begin{abstract}
The present study deal with constructing an analytical model within Hamiltonian formulation to design invariant relative orbits due to the perturbation of $\mathrm{J}_{2}$ and the lunisolar attraction. To fade the secular drift separation over the time between two neighboring orbits, two second order conditions that guarantee that drift are derived and enforced to be equal.
\end{abstract}

Keywords: Formation Flying, Invariant Relative Orbits, Secular Drift, Second Order Conditions, Neighboring Orbits, Lunisolar Attraction

\section{INTRODUCTION}

Within the last decades several projects have been started where the goal is to use multiple satellites working as one. Thus formation flying clusters is a relatively new concept in space science. The design of relative navigation and control systems of the spacecrafts in this cluster is certainly one of the most challenging topics. In order to ease the tasks of these subsystems, a proper reference trajectory must be conceived and a relative motion, which shows no drift even in presence of perturbation forces, could be a very attractive solution. Mentioned here the most important papers dealing with this problem, the dominant $\mathrm{J}_{2}$ oblateness effect in perturbations is considered as the main destroyer to the formation configuration and $\mathrm{J}_{2}$ invariant orbit is firstly defined by (Schaub and Alfriend, 2001) in mean orbital elements to minimize the amount of fuel to maintain. They introduced an analytical technique to derive a class of orbits that are termed 'invariant' orbits. Satellites placed into 'invariant' orbits are not immune to the disturbance force, but instead two satellites placed into these orbits will have the same drift and thus the cluster remains together. The 'invariant' orbits are created by using two first order conditions that determine the correct differences in semi-major axis, eccentricity and inclination. Zhang and Dai (2002) removed the drifts by adjusting the semi-axis of the follower satellite and obtained a similar conclusion. However, all the above works are based on the mean orbital elements instead of the Cartesian coordinates, which prevents the $\mathrm{J}_{2}$ invariant orbit from being applied. Biggs and Becerra (2005) proposed a method to determinate the $\mathrm{J}_{2}$ invariant orbit with the leader's orbit of zero inclination based on the targeting method in chaos dynamics. Subsequently, (Yan and Alfriend, 2006; Breger and How, 2006; Xu and Xu, 2007; Sabatini et al., 2009) investigated $\mathrm{J}_{2}$ invariant relative orbits from the perspective of relative orbital elements and relative Cartesian coordinates, thereby achieving different types of invariant conditions through numerical searches. El-Salam et al. (2006) used the Hamiltonian framework to construct an analytical method to design invariant relative constellation orbits due to the zonal harmonics; up to the second order, assuming $\mathrm{J}_{2}$ being of order 1. Rahoma and Metris (2012) constructed an analytical method using Hamiltonian framework to design invariant relative emphasized on secular oblateness perturbations due to the zonal harmonics $\mathrm{J}_{2}, \mathrm{~J}_{3}, \mathrm{~J}_{4}$ and the third body effect, assuming $\mathrm{J}_{2}$ being of order 1 .

After this introduction, the model of the oblateness of the Earth, truncated beyond the second zonal harmonic $\mathrm{J}_{2}$ and the lunisolar attractions is devoted. The Hamiltonian of the problem was constructed by considering $\square \mathrm{n}_{2}$; the mean motion of the Moon; as the small parameter of the problem. The normalized Hamiltonian of the system considered, F, after eliminating the short, intermediate and long period terms respectively using a perturbation technique based on Lie series, Lie transform and Bohlin's technique, is represented as (Radwan, 2002). The 
expressions for the time rate of change of the secular elements are obtained, second order conditions are established between the differences in moment an elements are equal on the average.

\subsection{Problem Formulate}

We consider the motion of artificial satellites in the Earth's gravity fields and perturbed by lunisolar attraction.

Let us assume, with sufficient accuracy, the following assumptions:

- The geopotential is restricted to the $\mathrm{J}_{2}$ part, like in the main problem of artificial satellite theory

- Sun and Moon move in circular orbits in the fixed ecliptic plane. Then we can set $\mathrm{u}_{2}=\mathrm{n}_{2} \mathrm{t}+$ const and $\mathrm{u}_{3}=\mathrm{n}_{3} \mathrm{t}+$ const, where $\left(\mathrm{u}_{2}, \mathrm{n}_{2}\right)$ and $\left(\mathrm{u}_{3}, \mathrm{n}_{3}\right)$ are the argument of latitude and the mean motion of Moon and Sun respectively. Noting that the time appears explicitly through $\mathrm{u}_{2}$ and $\mathrm{u}_{3}$, we augment the set by the addition of the pair $\mathrm{k}_{2}=\mathrm{n}_{2} \mathrm{t}+$ const, $\mathrm{k}_{3}=\mathrm{n}_{3} \mathrm{t}+$ const and their conjugates $\mathrm{K}_{2}$ and $\mathrm{K}_{3}$ respectively. Lunisolar potential are truncated at the second Legendre polynomial term.

\section{MATERIALS AND METHODS}

\subsection{The Normalized Hamiltonian}

Consideringn $_{2}$ as the small parameter of the problem, the orders of magnitude up to the second order of the involved parameters are defined as follows $\mathrm{n}_{2}=0.03 \equiv \cdot$. (Zero) he normalized Hamiltonian of the system considered, F, after eliminating the short, intermediate and long period terms respectively using a perturbation technique based on Lie series and Lie transform to eliminate the short and intermediate terms and Bohlin's technique to eliminate the long period terms can be represented in terms of Delaunay set as (Equation 1-4), (Radwan, 2002):

$\mathrm{F}=\sum_{\mathrm{s}=0}^{2} \frac{\varepsilon^{\mathrm{s}} \mathrm{s}}{\mathrm{s}} \mathrm{F}_{\mathrm{s}}$

Where:

$$
\begin{aligned}
& \mathrm{F}_{0}=\frac{\mu^{2}}{2 \mathrm{~L}^{2}}-\mathrm{K}_{2} \\
& \mathrm{~F}_{1}=\frac{\eta_{2}}{\mathrm{~L}^{2} \mathrm{G}^{3}}\left(-1+3\left(\frac{\mathrm{H}}{\mathrm{G}}\right)^{2}\right)+\mathrm{AK}_{3}
\end{aligned}
$$

where, $\mu=\mathrm{k}^{2} \mathrm{~m}_{0}$, is the gravitational const., $\mathrm{m}_{0}, \mathrm{~m}_{2}$ and $\mathrm{m}_{3}$ is the mass of Earth, Moon and Sun respectively $\mathrm{S}_{\mathrm{j}}=$ $\sin \mathrm{i}_{\mathrm{j}}$ and $\mathrm{C}_{\mathrm{j}}=\cos \mathrm{i}_{\mathrm{j}}, \mathrm{j}=2,3$ refer to Moon and Sun and $\mathrm{i}$ is the inclination:

$$
\eta_{2}=\frac{\mathrm{R}^{2} \mu^{4} J_{2}}{2 \mathrm{n}_{2}^{2}}, \mathrm{~A}=\frac{-\mathrm{n}_{3}}{\mathrm{n}_{2}^{2}}, \mathrm{~A}_{1}=\frac{9 \mathrm{k}^{2} \mathrm{~m}_{2}}{2 \mu^{2} \mathrm{n}_{2}^{4} \mathrm{a}_{2}^{3}}, \mathrm{~A}_{3}=\frac{9 \mathrm{k}^{2} \mathrm{~m}_{3}}{2 \mu^{2} \mathrm{n}_{2}^{4} \mathrm{a}_{3}^{4}}
$$

where, $\mathrm{R}$ is the radius of the Earth, $\alpha$ is the semi major axis of the satellite.

Using the Hamiltonian canonical equations of the motion, to write the secular drift in the argument of mean latitude $(\theta=1+\mathrm{g})$ :

$$
\theta=\frac{\partial \mathrm{F}}{\partial \mathrm{L}}+\partial \mathrm{G} \frac{\partial \mathrm{F}}{\partial \mathrm{G}}
$$

Yields:

$$
\begin{aligned}
& \theta=\frac{\mu^{2}}{L^{3}}+\varepsilon \frac{3 \eta_{2}}{G^{6} L^{4}}\left(G^{3}-3 G H^{2}+G^{2} L-5 H^{2} L\right)+\frac{\varepsilon^{2} 2 L}{2 ! 3 G^{3}} \\
& \left(\begin{array}{l}
A 1\left(\begin{array}{l}
6\left(1-\frac{\mathrm{H}^{2}}{\mathrm{G}^{2}}\right)^{-1 / 2}\left(2 \mathrm{G}^{2}-2 \mathrm{H}^{2}+\mathrm{GL}\right) \mathrm{G}^{2} \mathrm{HC}_{2} \mathrm{~S}_{2}-9 \mathrm{G}^{5}-3 \mathrm{G}^{2} \mathrm{H}^{2}- \\
9 \mathrm{G}^{4} \mathrm{~L}+10 \mathrm{G}^{3} \mathrm{~L}^{2}+30 \mathrm{~L}^{2} \mathrm{GH}^{2}-15 \mathrm{~L}^{3} \mathrm{H}^{2}+3\left(\mathrm{G}^{2}+\mathrm{GL}-10 \mathrm{~L}^{2}\right) \mathrm{G}^{3} \mathrm{~S}_{2}^{2}
\end{array}\right) \\
+\mathrm{A}_{3} \mathrm{G}^{3}\left(-42 \mathrm{G}^{2}-42 \mathrm{LG}+100 \mathrm{~L}^{2}+3\left(3 \mathrm{G}^{2}+3 \mathrm{GL}-10 \mathrm{~L}^{2} \mathrm{~S}_{3}^{2}\right)\right)
\end{array}\right)
\end{aligned}
$$

and the secular drift rates of the longitude of the ascending node:

$$
\stackrel{\square}{\mathrm{h}}=\frac{\partial \mathrm{F}}{\partial \mathrm{H}}
$$

Yields:

$$
\begin{aligned}
& \mathrm{h}=\varepsilon \frac{6 \eta_{2} \mathrm{H}}{\mathrm{G}^{5} \mathrm{~L}^{3}}+\frac{\varepsilon^{2} 2 \mathrm{~L}^{2}}{2 ! \mathrm{G}^{2}} \mathrm{~A}_{1} \\
& \left(2\left(1-\frac{\mathrm{H}^{2}}{\mathrm{G}^{2}}\right)^{-1 / 2}\left(\mathrm{G}^{2}-2 \mathrm{H}^{2}\right) \mathrm{GC}_{2} \mathrm{~S}_{2}-\mathrm{G}^{2} \mathrm{H}+5 \mathrm{~L}^{2} \mathrm{H}\right)
\end{aligned}
$$


Using an intermediary variable $\eta=\sqrt{1-\mathrm{e}^{2}}$, Equation 5-8 can be rewritten using Keplerian elements, as:

$$
\theta=-\frac{\mu^{2}}{L^{3}}+\varepsilon \frac{-9 \eta_{2}}{6 L^{7} \eta^{4}}(3+5 \cos 2 i+\eta(1+3 \cos 2 i))+\varepsilon^{2} L^{3}
$$$$
\left\{\begin{array}{l}
\frac{25}{3} \mathrm{~A}_{1}+\frac{100}{3} \mathrm{~A}_{3}-10 \mathrm{~A}_{1} \mathrm{~S}_{2}^{2}-10 \mathrm{~A}_{1} \mathrm{~S}_{2}^{2}-10 \mathrm{~A}_{3} \mathrm{~S}_{2}^{2}+ \\
5 \mathrm{~A}_{1} \cos 2_{\mathrm{i}}+\frac{1}{\eta}\left(\begin{array}{l}
-144+\mathrm{A}_{1}\left(-3+\mathrm{S}_{2}^{2}\right)+3 \mathrm{~A}_{3} \mathrm{~S}_{2}^{2} \\
+2 \mathrm{~A}_{1} \mathrm{C}_{2} \mathrm{~S}_{2} \frac{\cos \mathrm{i}}{\sin \mathrm{i}}
\end{array}\right) \\
+\eta\left(\frac{-5}{2} \mathrm{~A}_{1}-\frac{5}{2} \mathrm{~A}_{1} \cos 2 \mathrm{i}\right) \\
+\eta^{2}\left[\begin{array}{l}
\frac{-7}{2} \mathrm{~A}_{1}-14 \mathrm{~A}_{3}+\mathrm{A}_{1} \mathrm{~S}_{2}^{2}+3 \mathrm{~A}_{3} \mathrm{~S}_{2}^{2}-\frac{1}{2} \mathrm{~A}_{1} \cos 2 \mathrm{i} \\
+\mathrm{A}_{1} \mathrm{C}_{2} \mathrm{~S}_{2} \frac{\cos \mathrm{i}}{\sin \mathrm{i}}-\mathrm{A}_{1} \mathrm{C}_{2} \mathrm{~S}_{2} \frac{\cos 3 \mathrm{i}}{\sin \mathrm{i}}
\end{array}\right\}
\end{array}\right\}
$$

And:

$$
\begin{aligned}
& \mathrm{h}=\varepsilon \frac{12 \eta_{2}}{\mathrm{~L}^{7} \eta^{4}} \cos \mathrm{i}-2 \varepsilon^{2} \mathrm{~A}_{1} \mathrm{~L}^{3} \\
& \left\{\left(\frac{-5}{\eta}+\eta\right) \cos \mathrm{i}+2 \eta \mathrm{C}_{2} \mathrm{~S}_{2} \frac{\cos 2 \mathrm{i}}{\sin \mathrm{i}}\right\}
\end{aligned}
$$

To proscription two neighboring orbits from drifting apart, the average secular growth needs to be equal. Since the mean angle quantities $1, \mathrm{~g}$ and $\mathrm{h}$ do not directly contribute to the secular growth, their values can be chosen at will. However, the mean moment a values $L, G$ and $H$ (and therefore implicitly a, e and i) must be carefully chosen to match the secular drift rates. To hinder the satellites from drifting apart over time, it must be matched all three rates. We impose the condition that the relative average drift rate of the angle between the radius vectors be zero i.e.:

$$
\begin{aligned}
& \mathrm{h}_{\mathrm{i}}=\mathrm{h}_{\mathrm{j}} \forall \mathrm{i} \neq \mathrm{j} \\
& \hat{\theta}_{\mathrm{i}}=\theta_{\mathrm{j}} \forall \mathrm{i} \neq \mathrm{j}
\end{aligned}
$$

where, $\stackrel{\square}{\theta}$ and $\mathrm{h}$ can be rewritten as, Equation (11) and (12)

$$
\theta=-\frac{\mu^{2}}{\mathrm{~L}^{3}}+\sum_{\mathrm{n}=1}^{2} \sum_{\mathrm{m}=-4}^{2} \sum_{\mathrm{s}=0}^{3} \varepsilon^{\mathrm{n}} \mathrm{x}_{\theta}^{\mathrm{nmt}} \mathrm{L}^{10 \mathrm{n}-17} \eta^{\mathrm{m}} \cos (\mathrm{si}) \sin ^{\delta_{5}^{\theta}}(\mathrm{i})
$$

$$
\mathrm{h}=\sum_{\mathrm{n}=1}^{2} \sum_{\mathrm{m}=-4}^{2} \sum_{\mathrm{s}=0}^{3} \varepsilon^{\mathrm{n}} \mathrm{X}_{\mathrm{h}}^{\mathrm{nnt}} L^{10 \mathrm{n}-17} \eta^{\mathrm{m}} \cos (\mathrm{si}) \sin ^{\delta_{\mathrm{s}}^{\mathrm{h}}}(\mathrm{i})
$$

where, the non-vanishing coefficients $\mathrm{x}_{\theta}{ }^{\mathrm{nmt}}$ and $\mathrm{x}_{\mathrm{h}}{ }^{\mathrm{nmt}}$ are computed in Appendix I.

And:

$$
\delta_{\mathrm{S}}^{\theta}=\left[\begin{array}{cl}
-1 & \mathrm{~s}=\text { odd } \\
0 & \mathrm{~s}=\text { even },
\end{array} \delta_{\mathrm{s}}^{\mathrm{h}}=\left[\begin{array}{rl}
0 & \mathrm{~s}=\text { odd } \\
-1 & \mathrm{~s}=\text { even }
\end{array}\right.\right.
$$

\subsection{Second Order Expansions Conditions}

Using the second order expansions conditions described by (Rahoma and Metris, 2012) for $\theta_{i}$ and $\mathrm{h}_{\mathrm{i}}$ from the mean value denoted by 0 to write the drift rate about reference orbital elements:

$$
\begin{aligned}
& \delta \dot{\theta}_{\mathrm{i}}=\dot{\theta}_{\mathrm{i}}-\dot{\theta}_{0}=\left.\delta \mathrm{L} \frac{\partial \dot{\theta}_{\mathrm{i}}}{\partial \mathrm{L}}\right|_{\mathrm{x}=\mathrm{xo}}+\left.\delta \eta \frac{\partial \dot{\theta}_{\mathrm{i}}}{\partial \eta}\right|_{\mathrm{x}=\mathrm{xo}}+\left.\delta \mathrm{i} \frac{\partial \dot{\theta}_{\mathrm{i}}}{\partial \mathrm{i}}\right|_{\mathrm{x}=\mathrm{xo}}+\frac{1}{2 !} \\
& {\left[\begin{array}{l}
\left.(\delta \mathrm{L})^{2} \frac{\partial^{2} \dot{\theta}_{\mathrm{i}}}{\partial \mathrm{L}^{2}}\right|_{\mathrm{x}=\mathrm{xo}}+\left.(\delta \eta)^{2} \frac{\partial^{2} \dot{\theta}_{\mathrm{i}}}{\partial \eta^{2}}\right|_{\mathrm{x}=\mathrm{xo}}+\left.(\delta \mathrm{i})^{2} \frac{\partial^{2} \dot{\theta}_{\mathrm{i}}}{\partial \mathrm{i}^{2}}\right|_{\mathrm{x}=\mathrm{xo}} \\
\left.+\left.\delta \mathrm{L} \delta \eta \frac{\partial^{2} \dot{\theta}_{\mathrm{i}}}{\partial \mathrm{L} \partial \eta}\right|_{\mathrm{x}=\mathrm{xo}}+\left.\delta \eta \delta \mathrm{i} \frac{\partial^{2} \dot{\theta}_{\mathrm{i}}}{\partial \eta \partial_{\mathrm{i}}}\right|_{\mathrm{x}=\mathrm{xo}}+\left.\delta \mathrm{i} \delta \mathrm{L} \frac{\partial^{2} \dot{\theta}_{\mathrm{i}}}{\partial \mathrm{i} \partial \mathrm{L}}\right|_{\mathrm{x}=\mathrm{xo}}\right]_{\mathrm{v}}^{\mathrm{v}} \\
\delta \dot{\mathrm{h}}_{\mathrm{i}}=\dot{\mathrm{h}}_{\mathrm{i}}-\dot{\mathrm{h}}_{0}=\left.\delta \mathrm{L} \frac{\partial \dot{\mathrm{h}}_{\mathrm{i}}}{\partial \mathrm{L}}\right|_{\mathrm{x}=\mathrm{xo}}+\left.\delta \eta \frac{\partial \dot{\mathrm{h}}_{\mathrm{i}}}{\partial \eta}\right|_{\mathrm{x}=\mathrm{xo}}+\left.\delta \mathrm{i} \frac{\partial \dot{\mathrm{h}}_{\mathrm{i}}}{\partial \mathrm{i}}\right|_{\mathrm{x}=\mathrm{xo}}+\frac{1}{2 !} \\
\left.(\delta \mathrm{L})^{2} \frac{\partial^{2} \dot{\mathrm{h}}_{\mathrm{i}}}{\partial \mathrm{L}^{2}}\right|_{\mathrm{x}=\mathrm{xo}}+\left.(\delta \eta)^{2} \frac{\partial^{2} \dot{\mathrm{h}}_{\mathrm{i}}}{\partial \eta^{2}}\right|_{\mathrm{x}=\mathrm{xo}}+\left.(\delta \mathrm{i})^{2} \frac{\partial^{2} \dot{\mathrm{h}}_{\mathrm{i}}}{\partial \mathrm{i}^{2}}\right|_{\mathrm{x}=\mathrm{xo}} \\
+\left.\delta \mathrm{L} \delta \eta \frac{\partial^{2} \dot{\mathrm{h}}_{\mathrm{i}}}{\partial \mathrm{L} \partial \eta}\right|_{\mathrm{x}=\mathrm{xo}}+\left.\delta \eta \delta \mathrm{i} \frac{\partial^{2} \dot{\mathrm{h}}_{\mathrm{i}}}{\partial \eta \partial_{\mathrm{i}}}\right|_{\mathrm{x}=\mathrm{xo}}+\left.\delta \mathrm{i} \delta \mathrm{L} \frac{\partial^{2} \dot{\mathrm{h}}_{\mathrm{i}}}{\partial \mathrm{i} \partial \mathrm{L}}\right|_{\mathrm{x}=\mathrm{xo}}
\end{array}\right]}
\end{aligned}
$$

where, we make use of the fact that $\dot{\theta}=\dot{\theta}(L, \eta, i)$ and $\dot{\mathrm{h}}=\dot{\mathrm{h}}(\mathrm{L}, \eta, \mathrm{i})$ only, also supposing $\delta \theta$ that is the difference in mean latitude rates $\delta \mathrm{L}=\mathrm{L}_{\mathrm{i}}-\mathrm{L}_{0}, \delta \eta=\eta_{\mathrm{i}}-\eta_{0}, \delta_{\mathrm{i}}=\mathrm{i}_{\mathrm{i}}-$ $\mathrm{i}_{0}, \mathrm{x}=\mathrm{L}, \eta, \mathrm{i}$ and $\mathrm{x}_{0}=\mathrm{L}_{0}, \eta_{0}, \mathrm{i}_{0}$.

Note that this theory will lead to an analytical second order conditions on the mean orbit elements. To establish a more precise set of orbit elements satisfying Equation 9 and 10, either $\delta \mathrm{L}, \delta \eta$ or $\delta$ i could be chosen and the remaining two moment a orbit element differences found through a numerical root solving technique. 
The required derivatives can be evaluated as:

$$
\begin{aligned}
& \dot{\theta} \mathrm{L}=-\frac{3 \mu^{2}}{\mathrm{~L}^{4}}+\sum_{\mathrm{n}=1}^{2} \sum_{\mathrm{m}=-4}^{2} \sum_{\mathrm{s}=0}^{3} \varepsilon^{\mathrm{n}}(10 \mathrm{n}-17) \mathrm{x}_{\theta}^{\mathrm{nmt}} \mathrm{L}^{10 \mathrm{n}-18} \eta^{\mathrm{m}} \cos (\mathrm{si}) \sin ^{\delta_{5}^{\theta}}(\mathrm{i}) \\
& \dot{\mathrm{h}}_{\mathrm{L}}=\sum_{\mathrm{n}=1}^{2} \sum_{\mathrm{m}=-4 \mathrm{~s}=0}^{2} \sum^{3} \varepsilon^{\mathrm{n}}(10 \mathrm{n}-17) \mathrm{x}_{\mathrm{h}}^{\mathrm{nmt} t} \mathrm{~L}^{10 \mathrm{n}-18} \eta^{\mathrm{m}} \cos (\mathrm{si}) \sin ^{\delta^{\mathrm{h}}}(\mathrm{i}) \\
& \dot{\beta}_{\mathrm{n}}=\sum_{\mathrm{n}=1}^{2} \sum_{\mathrm{m}=-4}^{2} \sum_{\mathrm{s}=0}^{3} \varepsilon^{\mathrm{n}} \mathrm{mx}_{\beta}^{\mathrm{nmt}} \mathrm{L}^{10 \mathrm{n}-17} \eta^{\mathrm{m}-1} \cos (\mathrm{si}) \sin ^{\delta_{5}^{\beta}}(\mathrm{i}) \\
& \dot{\beta}_{\mathrm{i}}=\sum_{\mathrm{n}=1}^{2} \sum_{\mathrm{m}=-4}^{2} \sum_{\mathrm{s}=0}^{3} \varepsilon^{\mathrm{n}} \mathrm{x}_{\beta}^{\mathrm{nmt}} \mathrm{L}^{10 \mathrm{n}-17} \eta^{\mathrm{m}} \\
& \left(\delta_{\mathrm{s}}^{\beta} \cos (\mathrm{i}) \cos (\mathrm{si}) \sin ^{\delta_{\mathrm{s}}^{\beta}-1}(\mathrm{i})-\mathrm{s} \sin (\mathrm{si}) \sin ^{\delta_{\mathrm{s}}^{\beta}}(\mathrm{i})\right) \\
& \dot{\theta} \mathrm{LL}=\frac{-12 \mu^{2}}{\mathrm{~L}^{5}} \sum_{\mathrm{n}=1}^{2} \sum_{\mathrm{m}=-4}^{2} \sum_{\mathrm{s}=0}^{3} \\
& \varepsilon^{\mathrm{n}}(10 \mathrm{n}-17)(10 \mathrm{n}-18) \mathrm{X}_{\theta}^{\mathrm{nmt}} \mathrm{L}^{10 \mathrm{n}-19} \eta^{\mathrm{m}} \cos (\operatorname{si}) \sin ^{\delta_{\mathrm{s}}^{\mathrm{h}}}(\mathrm{i}) \\
& \dot{\mathrm{h} L L}=\sum_{\mathrm{n}=1}^{2} \sum_{\mathrm{m}=-4}^{2} \sum_{\mathrm{s}=0}^{3} \varepsilon^{\mathrm{n}} \mathrm{f}_{\mathrm{e}} \\
& (10 n-17)(10 n-18) X_{h}^{n m t} L^{10 n-19} \eta^{m} \cos (s i) \sin ^{\delta_{s}^{h}}(i)
\end{aligned}
$$$$
\dot{\beta}_{\eta \eta}=\sum_{\mathrm{n}=1}^{2} \sum_{\mathrm{m}=-4}^{2} \sum_{\mathrm{s}=0}^{3} \varepsilon^{\mathrm{n}} \mathrm{m}(\mathrm{m}-1) \mathrm{X}_{\beta}^{\mathrm{nmt}} \mathrm{L}^{10 \mathrm{n}-17} \eta^{\mathrm{m}-2} \cos (\mathrm{si}) \sin ^{\delta_{\mathrm{s}}^{\beta}}(\mathrm{i})
$$$$
\dot{\beta}_{\mathrm{ii}}=\sum_{\mathrm{n}=1}^{2} \sum_{\mathrm{m}=-4}^{2} \sum_{\mathrm{s}=0}^{3} \varepsilon^{\mathrm{n}} \mathrm{X}_{\beta}^{\mathrm{nnt}} \mathrm{L}^{10 \mathrm{n}-17} \eta^{\mathrm{m}}
$$$$
\left[\begin{array}{l}
\left(\delta_{\mathrm{s}}^{\beta}+\left(\delta_{\mathrm{s}}^{\beta}\right)^{2}-\frac{1}{2}\left(\mathrm{~s}^{2}+\left(\delta_{\mathrm{s}}^{\beta}\right)^{2}\right) \sin ^{2}(\mathrm{i}) \cos (\mathrm{si}) \sin ^{\delta_{\mathrm{s}}^{\beta}-2}(\mathrm{i})\right. \\
+\left(-\frac{1}{2}\left(\mathrm{~s}^{2}+\left(\delta_{\mathrm{s}}^{\beta}\right)^{2} \cos (\mathrm{si})-2 \mathrm{~s}^{\delta_{\mathrm{s}}^{\beta}} \cot (\mathrm{i}) \sin (\mathrm{si})\right) \sin ^{\delta_{\mathrm{s}}^{\beta}}(\mathrm{i})\right)
\end{array}\right]
$$$$
\dot{\beta}_{\mathrm{L \eta}}=\sum_{\mathrm{n}=1}^{2} \sum_{\mathrm{m}=-4}^{2} \sum_{\mathrm{s}=0}^{3} \varepsilon^{\mathrm{n}}(10 \mathrm{n}-17) \mathrm{X}_{\beta}^{\mathrm{nmt} t} \mathrm{~L}^{10 \mathrm{n}-18} \eta^{\mathrm{m}-1} \cos (\mathrm{si}) \sin ^{\delta_{\mathrm{s}}^{\beta}}(\mathrm{i})
$$$$
\dot{\beta}_{\mathrm{Li}}=\sum_{\mathrm{n}=1}^{2} \sum_{\mathrm{m}=-4}^{2} \sum_{\mathrm{s}=0}^{3} \varepsilon^{\mathrm{n}}(10 \mathrm{n}-17) \mathrm{X}_{\beta}^{\mathrm{nmt}} \mathrm{L}^{10 \mathrm{n}-18} \eta^{\mathrm{m}}
$$$$
\left(\delta_{\mathrm{s}}^{\beta} \cos (\mathrm{si}) \cos (\mathrm{si}) \sin ^{\delta_{\mathrm{s}}^{\beta}-1}(\mathrm{i})-\mathrm{s} \sin (\mathrm{si}) \sin ^{\delta_{\mathrm{s}}^{\beta}}(\mathrm{i})\right)
$$$$
\dot{\beta}_{\eta \mathrm{i}}=\sum_{\mathrm{n}=1}^{2} \sum_{\mathrm{m}=-4}^{2} \sum_{\mathrm{s}=0}^{3} \varepsilon^{\mathrm{n}} \mathrm{mx}_{\beta}^{\mathrm{nmt}} \mathrm{L}^{10 \mathrm{n}-17} \eta^{\mathrm{m}-1}
$$$$
\left(\delta_{\mathrm{s}}^{\beta} \cos (\mathrm{si}) \cos (\mathrm{si}) \sin _{\mathrm{s}}^{\delta^{\beta}-1}(\mathrm{i})-\mathrm{s} \sin (\mathrm{si}) \sin _{\mathrm{s}}^{\delta_{\mathrm{s}}^{\beta}}(\mathrm{i})\right)
$$

Where:

$$
\beta=\theta, h, \dot{y}_{x}=\frac{\partial \dot{y}}{\partial x} \text { and } \dot{y}_{x x}=\frac{\partial^{2} \dot{y}}{\partial x \partial x} \text { with } y=\beta, \theta, \text { h and } x=L, \eta, i
$$

To enforce equal drift rates $\dot{\theta}_{i}$ and $\dot{\mathrm{h}}_{\mathrm{i}}$ between neighboring orbits, we must set $\dot{\delta} \theta$ and $\delta$ h equal to zero in expanded Equation 13 and 14, yields:

$$
\begin{aligned}
& 0=\frac{1}{2} \dot{\theta}_{\mathrm{LL}}(\delta \mathrm{L})^{2}+\frac{1}{2} \dot{\theta} \mathrm{L}_{\eta} \delta \mathrm{L} \delta \eta+\frac{1}{2} \dot{\theta}_{\eta \eta}(\delta \eta)^{2}+\left(\dot{\theta}_{\mathrm{L}}+\frac{1}{2} \delta \dot{\theta}_{\mathrm{iL}}\right) \\
& \delta \mathrm{L}+\left(\dot{\theta}_{\eta}+\frac{1}{2} \delta \mathrm{i} \dot{\theta}_{\eta \mathrm{i}}\right) \delta \eta+\left(\dot{\theta}_{\mathrm{i}}+\frac{1}{2} \delta \dot{\mathrm{i}}_{\mathrm{ii}}\right) \delta \mathrm{i} \\
& 0=\frac{1}{2} \dot{\mathrm{h}}_{\mathrm{LL}}(\delta \mathrm{L})^{2}+\frac{1}{2} \dot{\mathrm{h}}_{\mathrm{L \eta}} \delta \mathrm{L} \delta \eta+\frac{1}{2} \dot{\theta}_{\eta \eta}(\delta \eta)^{2}+\left(\dot{\mathrm{h}}_{\mathrm{L}}+\frac{1}{2} \delta \mathrm{i} \dot{\mathrm{h}}_{\mathrm{iL}}\right) \\
& \delta \mathrm{L}+\left(\dot{\mathrm{h}}_{\eta}+\frac{1}{2} \delta \mathrm{i} \dot{\theta}_{\eta \mathrm{i}}\right) \delta \eta+\left(\dot{\mathrm{h}}_{\mathrm{i}}+\frac{1}{2} \delta \mathrm{i} \dot{\mathrm{h}}_{\mathrm{ii}}\right) \delta \mathrm{i}
\end{aligned}
$$

Equation (15) and (16) are two simultaneous nonlinear algebraic equations in three unknowns, namely $\delta \mathrm{L}, \delta \eta, \delta \mathrm{i}$. When one of these three unknowns is assumed known (say $\delta i)$, these two equations can be solved as:

Multiplying Equation 15 by $\frac{1}{2} \dot{\mathrm{h}}_{\mathrm{LL}}$ and Equation 16 by $\frac{1}{2} \dot{\theta}_{\mathrm{LL}}$ and then subtracting yields:

$$
\delta \mathrm{L}=\frac{\mathrm{c}_{2}(\delta \eta)^{2}+\mathrm{c}_{4} \delta \eta+\mathrm{c}_{5}}{\mathrm{c}_{1} \delta \eta+\mathrm{c}_{3}}
$$

Where:

$$
\begin{aligned}
& \mathrm{c}_{1}=\frac{1}{2}\left(\dot{\theta}_{\mathrm{L \eta}} \dot{\mathrm{h}}_{\mathrm{LL}}-\dot{\theta}_{\mathrm{LL}} \dot{\mathrm{h}}_{\mathrm{L \eta}}\right) \\
& \mathrm{c}_{2}=\frac{1}{2}\left(\dot{\theta}_{\eta \eta} \dot{\mathrm{h}}_{\mathrm{LL}}-\dot{\theta}_{\mathrm{LL}} \dot{\mathrm{h}}_{\eta \eta}\right) \\
& \mathrm{c}_{3}=\left(\dot{\theta}_{\mathrm{L}} \frac{1}{2} \dot{\theta}_{\mathrm{iL}} \delta \mathrm{i}\right) \dot{\mathrm{h}}_{\mathrm{LL}}-\left(\dot{\mathrm{h}}_{\mathrm{L}}+\frac{1}{2} \dot{\mathrm{h}}_{\mathrm{iL}} \delta \mathrm{i}\right) \dot{\theta}_{\mathrm{LL}} \\
& \mathrm{c}_{4}=\left(\dot{\theta}_{\eta} \frac{1}{2} \dot{\theta}_{\eta \mathrm{i}} \mathrm{\delta i}\right) \dot{\mathrm{h}}_{\mathrm{LL}}-\left(\dot{\mathrm{h}}_{\eta}+\frac{1}{2} \dot{\mathrm{h}}_{\eta \mathrm{i}} \delta \mathrm{i}\right) \dot{\theta}_{\mathrm{LL}} \\
& \mathrm{c}_{5}=\left[\left(\dot{\theta}_{\mathrm{i}} \frac{1}{2} \dot{\theta}_{\mathrm{ii}} \delta \mathrm{i}\right) \dot{\mathrm{h}}_{\mathrm{LL}}-\left(\dot{\mathrm{h}}_{\mathrm{i}}+\frac{1}{2} \dot{\mathrm{h}}_{\mathrm{ii}} \delta \mathrm{i}\right) \dot{\theta}_{\mathrm{LL}}\right] \delta \mathrm{i}
\end{aligned}
$$

Substituting Equation 17 into Equation 15 yields an algebraic equation of fourth degree in $\delta \eta$ only in the form: 


$$
\mathrm{d}_{1}(\delta \eta)^{4}+\mathrm{d}_{2}(\delta \eta)^{3}+\mathrm{d}_{3}(\delta \eta)^{2}+\mathrm{d}_{4} \delta \eta+\mathrm{d}_{\mathrm{s}}=0
$$

Where:

$$
\begin{gathered}
\mathrm{d}_{1}=\mathrm{c}_{2}^{2} \dot{\theta}_{\mathrm{LL}}-\mathrm{c}_{1} \mathrm{c}_{2} \dot{\theta}_{\mathrm{L \eta}}+\mathrm{c}_{1}^{2} \dot{\theta} \eta \eta \\
\mathrm{d}_{2}=2 \mathrm{c}_{2} \mathrm{c}_{4} \dot{\theta}_{\mathrm{LL}}-\left(\mathrm{c}_{2} \mathrm{c}_{3}+\mathrm{c}_{4} \mathrm{c}_{1}\right) \dot{\theta}_{\mathrm{L \eta}}-\mathrm{c}_{1} \mathrm{c}_{2}\left(\dot{\theta}_{\mathrm{L}}+\frac{1}{2} \dot{\theta}_{\mathrm{iL}} \delta \mathrm{i}\right) \\
+\mathrm{c}_{1}^{2}\left(\frac{1}{2} \dot{\theta}_{\eta \mathrm{i}} \delta \mathrm{i}\right)+2 \mathrm{c}_{1} \mathrm{c}_{3} \dot{\theta}_{\eta \eta} \\
\mathrm{d}_{3}=\left(\mathrm{c}_{4}^{2} 2 \mathrm{c}_{2} \mathrm{c}_{5}\right) \dot{\theta}_{\mathrm{LL}}-\left(\mathrm{c}_{4} \mathrm{c}_{3}+\mathrm{c}_{5} \mathrm{c}_{1}\right) \dot{\theta}_{\mathrm{L \eta}}+\mathrm{c}_{3}^{2} \dot{\theta}_{\eta \eta}-\left(\mathrm{c}_{2} \mathrm{c}_{3}+\mathrm{c}_{4} \mathrm{c}_{1}\right) \\
\left(\dot{\theta}_{\mathrm{L}}+\frac{1}{2} \dot{\theta}_{\mathrm{iL}} \delta \mathrm{i}\right)+2 \mathrm{c}_{1} \mathrm{c}_{3}\left(\dot{\theta}_{\eta}+\frac{1}{2} \dot{\theta}_{\eta \mathrm{i}} \delta \mathrm{i}\right)+\mathrm{c}_{1}^{2}\left(\dot{\theta}_{\mathrm{i}}+\frac{1}{2} \dot{\theta}_{\mathrm{ii}} \delta \mathrm{i}\right) \delta \mathrm{i} \\
\mathrm{d}_{4}=2 \mathrm{c}_{4} \mathrm{c}_{\mathrm{s}} \dot{\theta}_{\mathrm{LL}}-\mathrm{c}_{\mathrm{s}} \mathrm{c}_{3} \dot{\theta}_{\mathrm{L \eta}}-\left(\mathrm{c}_{4} \mathrm{c}_{3}+\mathrm{c}_{5} \mathrm{c}_{1}\right)\left(\dot{\theta}_{\mathrm{L}}+\frac{1}{2} \dot{\theta}_{\mathrm{iL}} \delta \mathrm{i}\right) \\
+\mathrm{c}_{3}^{2}\left(\dot{\theta}_{\eta} \frac{1}{2} \dot{\theta}_{\eta \mathrm{i}} \delta \mathrm{i}\right)+2 \mathrm{c}_{1} \mathrm{c}_{3}\left(\dot{\theta}_{\mathrm{i}} \frac{1}{2} \dot{\theta}_{\mathrm{iij}} \delta \mathrm{i}\right) \delta \mathrm{i} \\
\mathrm{d}_{5}=\mathrm{c}_{5}^{2} \dot{\theta}_{\mathrm{LL}}-\mathrm{c}_{5} \mathrm{c}_{3}\left(\dot{\theta}_{\mathrm{L}}+\frac{1}{2} \dot{\theta}_{\mathrm{iL}} \delta \mathrm{i}\right)+\mathrm{c}_{3}^{2}\left(\dot{\theta}_{\mathrm{i}}+\frac{1}{2} \dot{\theta}_{\mathrm{ii}} \delta \mathrm{i}\right) \delta \mathrm{i}
\end{gathered}
$$

\section{RESULTS AND DISCUSSION}

\subsection{Solution of the Quartic Equation 18}

The roots of the quartic Equation 18 can be written as:

$$
(\delta \eta)_{1,2}=\mathrm{a}+\mathrm{b} \pm \mathrm{c},(\delta \eta)_{3,4}=\mathrm{a}+\mathrm{b} \pm \mathrm{d}
$$

Where:

$$
\begin{aligned}
& \mathrm{a}=-\frac{\mathrm{d}_{2}}{4 \mathrm{~d}_{1}}, \mathrm{~b}=\frac{1}{2} \sqrt{2 \mathrm{e}-\mathrm{f}-\mathrm{g}-\mathrm{h},} \\
& \mathrm{c}=\frac{1}{2} \sqrt{\mathrm{e}+\mathrm{f}+\mathrm{g}, \mathrm{d}}=\frac{1}{2} \sqrt{2 \mathrm{e}-\mathrm{f}-\mathrm{g}-\mathrm{h}}
\end{aligned}
$$

With:

$$
e=4 a^{2}-\frac{2 d_{3}}{3 d_{1}}, f=\frac{\sqrt[3]{2} i}{3 d_{1} j}, g=\frac{k}{3 * \sqrt[3]{2 d_{1}}}, h=\frac{1}{8 b}
$$

Where:

$$
\begin{aligned}
& i=d_{3}^{2}-3 d_{2} d_{4}+12 d_{1} d_{5}, j=\left(m+\sqrt{-4 i^{3}+m^{2}}\right) \\
& 1=-\left(\frac{d_{2}}{d_{1}}\right)^{3}+\frac{4 d_{2} d_{3}}{d_{1}^{2}}-\frac{4 d_{4}}{d_{1}}
\end{aligned}
$$

And:

$$
m=2 d_{3}^{3}-9 d_{2} d_{3} d_{4}+27 d_{1} d_{4}^{3}+27 d_{2}^{3} d_{5}-72 d_{1} d_{3} d_{5}
$$

Substituting the four roots $\delta \eta$ 's into Equation 17 yields the four constraints $\delta \mathrm{L}$ 's that guarantee the invariance of the relative motion of certain satellite constellation:

$$
\begin{aligned}
& (\delta \mathrm{L})_{1,2}=-\frac{\mathrm{C}_{2}\left(\mathrm{a}^{2}+\mathrm{b}^{2}+\mathrm{c}^{2}-2 \mathrm{ab} \pm 2 \mathrm{bc} \mp 2 \mathrm{ac}\right)+\mathrm{c}_{4}(\mathrm{a}-\mathrm{b}-\mathrm{c})+\mathrm{c}_{5}}{\mathrm{c}_{1}(\mathrm{a}-\mathrm{b}-\mathrm{c})+\mathrm{c}_{3}} \\
& (\delta \mathrm{L})_{3,4}=-\frac{\mathrm{C}_{2}\left(\mathrm{a}^{2}+\mathrm{b}^{2}+\mathrm{d}^{2}+2 \mathrm{ab} \mp 2 \mathrm{bd} \mp 2 \mathrm{ad}\right)+\mathrm{c}_{4}(\mathrm{a}+\mathrm{b}-\mathrm{d})+\mathrm{c}_{5}}{\mathrm{c}_{1}(\mathrm{a}+\mathrm{b}-\mathrm{d})+\mathrm{c}_{3}}
\end{aligned}
$$

\section{CONCLUSION}

This study introduced an approach to optimizing $\mathrm{J}_{2}$ and lunisolar attraction invariance between spacecraft that explicitly minimized the fuel use required to achieve the invariant states. This approach also allowed weights to be assigned the emphasis on invariance (i.e., preventing drift), minimizing fuel use and maintaining a desired geometry. In a formation where the principle control objective is to "not drift," the proposed approach could be used as a fuel-optimized formation flying control algorithm.

\section{REFRENCEES}

Biggs, J.D. and V.M. Becerra, 2005. A search for invariant relative satellite motion. Proceedings of the 4th Workshop on Satellite Constellations and Formation Flying, (SCFF' 05), Sao Jose dos Campos, Brazil, pp: 203-213.

Breger, L. and P. How, 2006. Partial-invariance for spacecraft formations. Proceedings of the AIAA/AAS Astrodynamics Conference, (AC' 06), Keystone, AIAA, pp: 65-85.

El-Salam, F.A.A., I.A. El-Tohamy, M.K. Ahmed, W.A. Rahoma and M.A. Rassem, 2006. Invariant relative orbits for satellite constellations: A second order theory. Appli. Math. Comput., 181: 6-20. DOI: 10.1016/j.amc.2006.01.004

Radwan, M., 2002. Resonance caused by the luni-solar attractions on a satellite of the oblate earth. Astrophysics Space Sci., 282: 551-562. DOI: 10.1023/A:1020971705850

Rahoma, W.A. and M. Metris, 2012. Invariant relative orbits taking into account third-body perturbation. Appli. Math., 3: 113-120. DOI: 10.4236/am.2012.32018 
Sabatini, M., D. Izzo and G.B. Palmerini, 2009. Minimum control for spacecraft formations in a $\mathrm{J} 2$ perturbed environment. Cel. Mech. Dynl. Astron. 105: 141-157. DOI: 10.1007/s10569-009-9214-5

Schaub, H. and K. Alfriend, 2001. $\mathrm{J}_{2}$ Invariant relative orbits for spacecraft formations. Celestial Mech. Dyn. Astron., 79: 77-95. DOI: 10.1023/A:1011161811472

$\mathrm{Xu}, \mathrm{M}$. and S.J. Xu, 2007. $\mathrm{J}_{2}$ Invariant relative orbits via differential correction algorithm. Acta Mech. Sin., 23: 585-595. DOI: 10.1007/s10409-007-0097-y
Yan, H. and K. Alfriend, 2006. Numerical searches and optimal control of invariant orbits. Proceedings of the 16th Annual AAS/AIAA Spaceflight Mechanics Meeting, (SMM' 06), AAS, Tampa, pp: 160-163.

Zhang, Y. and J. Dai, 2002. Satellite formation flying with perturbation. J. Nat. Univ. Defense Technol., 24: 6-10. 